University of South Carolina

Scholar Commons

$9-1-2021$

\title{
A CdZnTeSe Gamma Spectrometer Trained by Deep Convolutional Neural Network for Radioisotope Identification
}

\author{
Sandeep K. Chaudhuri \\ University of South Carolina - Columbia \\ Joshua W. Kleppinger \\ University of South Carolina - Columbia \\ Ritwik Nag \\ University of South Carolina - Columbia \\ Kaushik Roy \\ University of South Carolina - Columbia \\ Roijiba thanład additional works at: https://scholarcommons.sc.edu/aii_fac_pub \\ 'zriversity of South Carolina - Columbia \\ Part of the Computer Engineering Commons, and the Electrical and Computer Engineering Commons
}

See next page for additional authors

Reprinted from Hard X-Ray, Gamma-Ray, and Neutron Detector Physics XXIII, Volume 11838, 2021, pages 1183806-.

Copyright 2021 Society of Photo-Optical Instrumentation Engineers (SPIE). One print or electronic copy may be made for personal use only. Systematic reproduction and distribution, duplication of any material in this publication for a fee or for commercial purposes, and modification of the contents of the publication are prohibited.

Sandeep K. Chaudhuri, Joshua W. Kleppinger, Ritwik Nag, Kaushik Roy, Rojina Panta, Forest Agostinelli, Amit Sheth, Utpal N. Roy, Ralph B. James, \& Krishna C. Mandal. "A CdZnTeSe gamma spectrometer trained by deep convolutional neural network for radioisotope identification," Proc. SPIE Volume 11838, Hard X-Ray, Gamma-Ray, and Neutron Detector Physics XXIII, 1183806 (2021). https://doi.org/10.1117/ 12.2596456

This Article is brought to you by the Artificial Intelligence Institute at Scholar Commons. It has been accepted for inclusion in Publications by an authorized administrator of Scholar Commons. For more information, please contact digres@mailbox.sc.edu. 


\section{Author(s)}

Sandeep K. Chaudhuri, Joshua W. Kleppinger, Ritwik Nag, Kaushik Roy, Rojina Panta, Forest Agostinelli, Amit Sheth, Utpal N. Roy, Ralph B. James, and Krishna C. Mandal 


\section{A CdZnTeSe gamma spectrometer trained by deep convolutional neural network for radioisotope identification}

Chaudhuri, Sandeep, Kleppinger, Joshua, Nag, Ritwik, Roy, Kaushik, Panta, Rojina, et al.

Sandeep K. Chaudhuri, Joshua W. Kleppinger, Ritwik Nag, Kaushik Roy, Rojina Panta, Forest Agostinelli, Amit Sheth, Utpal N. Roy, Ralph B. James, Krishna C. Mandal, "A CdZnTeSe gamma spectrometer trained by deep convolutional neural network for radioisotope identification," Proc. SPIE 11838, Hard X-Ray, Gamma-Ray, and Neutron Detector Physics XXIII, 1183806 (1 September 2021); doi: 10.1117/12.2596456

Event: SPIE Optical Engineering + Applications, 2021, San Diego, California, United States 


\title{
A CdZnTeSe gamma spectrometer trained by deep convolutional neural network for radioisotope identification
}

\author{
Sandeep K. Chaudhuri ${ }^{\mathrm{a}}$, Joshua W. Kleppinger ${ }^{\mathrm{a}}$, Ritwik Nag ${ }^{\mathrm{a}}$, Kaushik Roy ${ }^{\mathrm{b}}$, Rojina Panta ${ }^{\mathrm{b}}$, Forest \\ Agostinellib $^{\mathrm{b}}$, Amit Sheth ${ }^{\mathrm{b}}$, Utpal N. Roy ${ }^{\mathrm{c}}$, Ralph B. James ${ }^{\mathrm{c}}$, Krishna C. Mandal ${ }^{\mathrm{a}^{*}}$ \\ ${ }^{a}$ Department of Electrical Engineering, University of South Carolina, Columbia, SC 29208, USA; \\ ${ }^{\mathrm{b}}$ Artificial Intelligence Institute, Department of Computer Science and Engineering, University of \\ South Carolina, Columbia, SC 29208, USA; 'Savannah River National Laboratory, Science, \\ Engineering and Energy Directorate, Aiken, SC 29831, USA
}

\begin{abstract}
We present a novel machine learning (ML) approach for fast and accurate identification of gamma-ray energies using room-temperature semiconductor detectors. Unlike conventional ML techniques, which uses pulse height spectra to train pattern recognition algorithms, the present ML approach has been trained with raw preamplifier pulses. Identification of radioisotopes through gamma spectroscopy has immense application in the field of radioactive waste characterization, in situ monitoring of nuclear reactors and spent nuclear fuel storage casks, homeland security, and environmental geology. Conventional spectroscopic methods relying on peak search and match, template matching, and regions of interest are not particularly effective because of the significant variation of radiological background based on immediate surroundings, attenuation in shielding materials, variation in position and geometry of detectors, and limitations in acquisition of statistically adequate data. We report the development of a deep convolutional neural network (CNN) which can identify gamma-ray energies in extremely short duration. The $\mathrm{CNN}$ has been trained using simulated data that resembles charge pulses produced by CdZnTeSe (CZTS) detector, an emerging and novel room-temperature gamma detector. A MonteCarlo simulation program has been designed in-house for generating the pulses. The CNN has been trained on the equivalent of $90 \%$ of the simulated data, and it has been validated on $10 \%$ of simulated data. Percentage error as low as $0.3 \%$ in the prediction of the gamma rays has been demonstrated and that too within detection time of 0.28 milli-sec. The effect of variation of detector resolution on the performance of the CNN has also been investigated.
\end{abstract}

Keywords: Room-temperature $\gamma$-ray detection, deep convolutional neural network (CNN), machine learning (ML), semiconductor radiation detector, wide bandgap semiconductor, CdZnTeSe (CZTS)

\section{INTRODUCTION}

Detection and identification of nuclear isotopes through gamma-ray detection is a conventional approach and has been in use for a long time [1] - [3]. The gamma-ray energies are signatures of transitions of excited nuclei to their ground states which can be used to determine the identity of the isotope. The gamma rays being highly penetrating in nature are easy to detect even through reasonably thick layers of shielding such as soil, concrete, and stainless steel. Ironically, the penetrating nature of gamma rays makes it difficult to stop them completely in detectors for efficient detection. Hence, special detector materials with high density and high atomic number constituents are chosen for gamma-ray detection. Sensitive applications of radiation detection for homeland security and border protection involve identification of nuclear isotopes, monitoring and safeguard of special nuclear materials (SNM) and spent nuclear fuels (SNF), monitoring soil and ground water contamination during nuclear fallout or accidental leakage in nuclear storage sites, etc. [4] - [9]. Geophysical applications include measurement of variation of radon concentrations in soil or groundwater through gamma-ray detection to predict earthquakes [10], [11]. Nuclear physics experiments involving nuclear reactions use gamma-ray detection to identify the daughter products and their excited states [12].

There are many practical challenges when it comes to field deployment of gamma-ray detectors. The energy resolution of the detection system is a major factor for accurate determination of gamma-ray energies. Detectors with lower resolution often produce overlapping peaks in the detection spectrum making it difficult to resolve the actual energies. Higher resolution gamma-ray detectors such as $\mathrm{HPGe}$ or $\mathrm{Si}(\mathrm{Li})$ on the other hand involve bulky cryogenic attachments for their

*mandalk@cec.sc.edu; phone+1 803 777-2722.

Hard X-Ray, Gamma-Ray, and Neutron Detector Physics XXIII, edited by Nerine J. Cherepy

Michael Fiederle, Ralph B. James, Proc. of SPIE Vol. 11838, 1183806 · ㄷ 2021 SPIE

CCC code: $0277-786 \mathrm{X} / 21 / \$ 21 \cdot$ doi: $10.1117 / 12.2596456$ 
proper functioning. The development of compound semiconductor detectors such as CdTe, CdZnTe (CZT), CdZnTeSe (CZTS), $\mathrm{HgI}_{2}, \mathrm{CsPbBr}_{3}$ are room-temperature detectors that can provide sufficiently high energy resolution at roomtemperature and with a compact form factor [13] - [21]. Among these detectors CdZnTeSe or CZTS, a novel quaternary wide bandgap semiconductor, stands out in terms of superior energy resolution and low cost of production [22] - [29]. Other challenges common to any kind of detection system involve the presence of excessive background radiation from natural or artificial sources in the vicinity, dependence of detector sensitivity, and efficiency on the source-detector geometry involving their relative distance and angular position.

Due to the sensitive nature of the field applications and often due to the presence of high radiation levels that can damage the detector and the associated read-out electronics, the duration of exposure of the detector assembly to the source are often minimized. However, accurate determination of gamma-ray energies needs a fully evolved detection spectrum, which might require data acquisition time lasting hours depending on the source strength, distance between the source and detector, etc. Although the identification of a peak in a detection spectrum can be achieved using simple algorithms based on peak detection (energy) and matching to a standard built-in library [1], [2], [30] - [32], trained spectroscopists are needed to identify the region-of-interest in the pulse-height spectra (PHS) in the first place. Recently, machine learning (ML) approaches are being used extensively for automated detection and spectrometric analysis, which not only minimizes human intervention but also are incredibly fast [33] - [37]. ML approaches can identify peaks using data acquired for a short duration even for detectors with poor energy resolution [38]. ML approaches relies primarily on pattern recognition rather than individual peak analysis. In supervised ML approaches a model is automatically developed through training on an adequate number of solved examples. Intensive computations involving human interventions are required only during the training purposes. A wide variety of automated and fundamental pattern recognition techniques such as Bayesian classifiers and discriminant functions [39], artificial neural networks (ANN) [40], methods for the induction of decision trees [41] and hybrid techniques combining the useful features of the above techniques such as the abductory induction mechanism (AIM) [42] and adaptive learning network (ALN) techniques [33] has already been reported.

In this work we present a fundamentally different machine learning approach that involves one-dimensional pattern recognition based on supervised learning using raw preamplifier pulses instead of fully developed detection spectra. This approach has all the flexibility of digital spectroscopy such as offline manipulation of pulses and pulse shapes even after the data have been acquired. Additionally, the approach does not require expensive hardware such as a spectroscopy amplifier and multi-channel analyzer. We have trained a deep convolutional neural network to predict gamma-ray energies using simulated data that resemble preamplifier charge pulses obtained using a CZTS detector fabricated in our laboratory. The method demonstrates the efficiency of the trained spectrometer by validating it using simulated data. The dependence of the prediction efficiency of the trained $\mathrm{CNN}$ on gamma-ray energies and detector resolution has also been investigated.

\section{OVERVIEW OF RADIATION DETECTION AND MACHINE LEARNING}

To reach a common accord of understanding among the readers of the two widely varying fields of radiation detection and artificial intelligence, we have defined and summarized the basics for the two topics below. The commonly used terminology followed in both the fields and those that have been used in this article are described below.

\subsection{Radiation detection and pulse-height spectroscopy (PHS)}

The basic process of gamma-ray detection involves a gamma-ray detector that interacts with the incoming gamma-rays emitted by a radioactive source to generate charge pairs within the detector material. The number of charge pairs produced is directly proportional to the energy of the incident radiation. The charge pairs drift under the influence of an electric field established due to a potential difference or bias applied across the detector. The drifting charges forms an electric current, which constitutes the detector signal. The detector signal is converted into a voltage signal using a preamplifier. Depending on the preamplifier type, the voltage output is either proportional to the input current or input charge. For gamma-ray spectroscopy a charge sensitive preamplifier is commonly used to obtain a voltage output proportional to the amount of charge produced in the detector due to the radiation interaction. The amplitude of the output voltage pulses, or what is referred to as pulse-height, is proportional to the input charge and hence the incident gamma energy. Ideally, the determination of the pulse heights from the preamplifier charge pulses is insensitive to the embedded noise in the preamplifier signal. Figure 1(a) shows a typical voltage pulse from a preamplifier output, which demonstrates the uncertainty in the pulse-height measurements due to the presence of noise in the detector signal. To determine accurately the pulse-height, the preamplifier pulses are filtered to increase the signal-to-noise ratio (SNR). The filtering is achieved 
using a spectroscopy amplifier. A spectroscopy amplifier uses a chain of low- and high-frequency filters to increase the SNR. The process also modifies the shape of the pulse in the process. Figure 1(b) shows such a shaped pulse using a shaping amplifier which uses a differentiator (CR) circuit followed by four integrator (RC) circuits (CR-RC ${ }^{4}$ ). The SNR can be seen to improve significantly. The shaped pulses are then fed into a multichannel analyzer (MCA), which uses comparator circuits to determine the pulse-height voltages and bin them in different channels to form a pulse-height spectrum (PHS). A higher amplitude for the pulse registers as a higher channel number in the MCA. The amplitude of the shaped pulses is proportional to the incident gamma-ray energy; thus, each channel represents an energy that can be determined through an appropriate calibration procedure. Gamma rays of different energies form separate peaks in the PHS at different channel numbers. Figure 1(c) shows a typical gamma PHS obtained using a typical analog spectrometer depicted in Fig. 1(d). The widths of the photo peak define the energy resolution of the spectrometer, which depends on the collective uncertainties attributed to the statistical nature of the gamma-photon generation, photon-detector interaction, charge transport within the detector material, and the electronic noise in the detection electronics. More details on radiation detection and measurements can be found elsewhere [43], [44]. The above discussion describes an analog pulse-height spectrometer. A digital spectrometer, as has been used for the present work, directly digitize each preamplifier outputpulses and stores the data in a PC, which can be analyzed and processed offline.
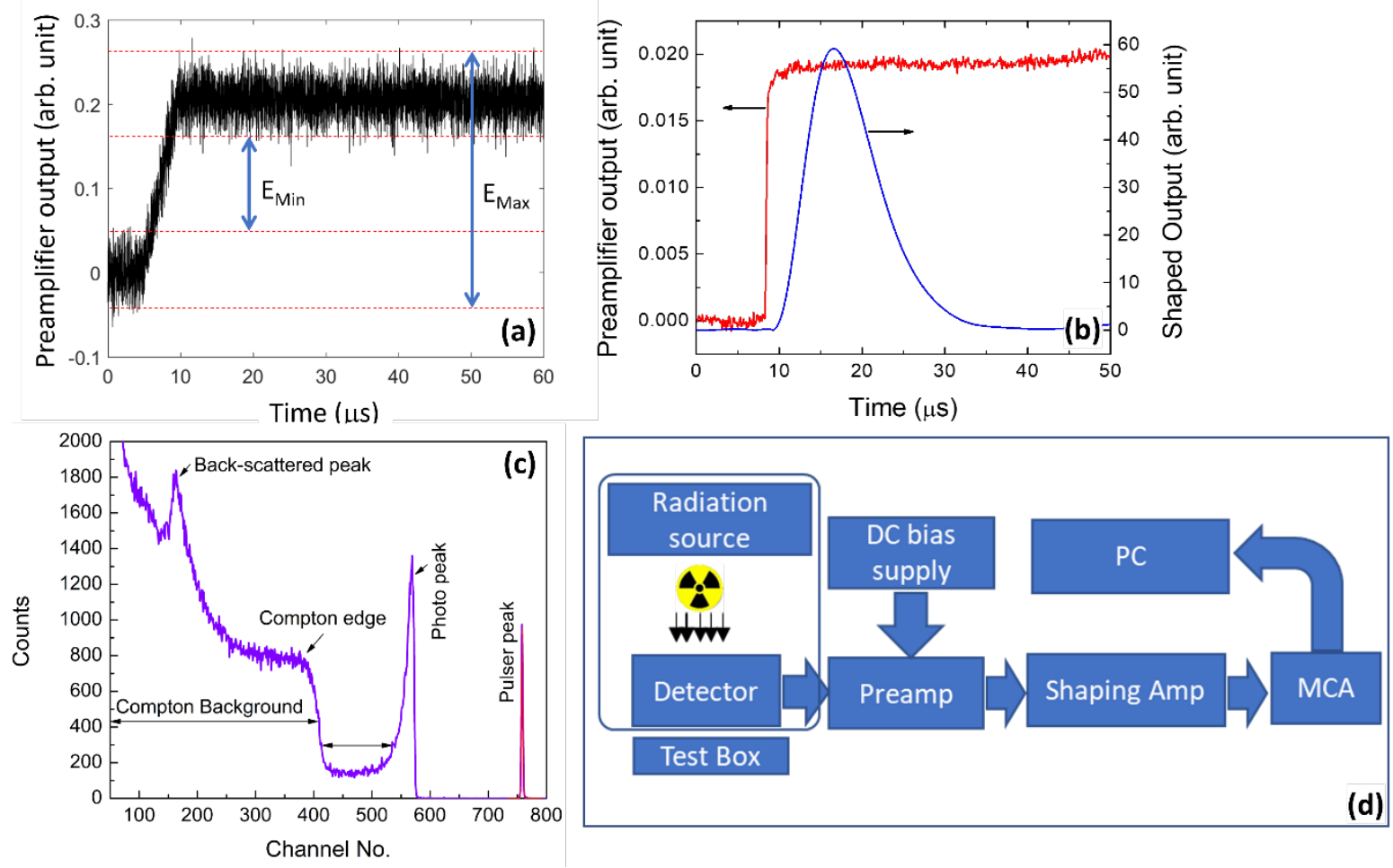

Figure 1. (a) A charge pulse obtained from a preamplifier output. Note the uncertainty in the pulse-height measurement due to the difference in $\mathrm{E}_{\max }$ and $\mathrm{E}_{\min }$. (b) A preamplifier pulse and a shaped pulse. Note how smooth the shaped pulse is. (c) A typical gamma pulse-height spectrum (PHS) for a Cs-137 source. (d) Schematic of a typical analog pulse-height spectrometer.

\subsection{Gamma-ray detection}

Gamma-ray detection is fundamentally different from charge particles due to the way gamma photons interact with matter. Charge particles interact with the target material atom primarily through Coulombic interactions. They gradually lose their kinetic energy through successive interactions with the electrons and the nuclei to eventually stop at a depth usually known as the projected range. Although the charge particles lose their energy gradually along the traversed path, the maximum energy is deposited near the end of the range. The energy lost is manifested as production of electron-hole pairs in semiconductors. It is obvious that the charge pairs are created all along the path traversed by the charged particle. Gamma (or x-ray) photons on the other hand, being electrically neutral, have different mechanisms of energy loss in a material 
medium. The primary interaction processes are elastic and inelastic scatterings. Elastic scattering leads to complete energy deposition by the incident photon which leads to the production of electron-hole pairs. This class of interactions are referred to as photoelectric (PE) events. Inelastic scattering on the other hand leads to Compton events where the incident photon imparts a fraction of its energy to the interacting atom. The amount of energy deposited by the incident photon depends on the angle of scattering $\Theta$. The maximum energy will be deposited in case of a head-on collision with the photon backscattering $\left(\Theta=180^{\circ}\right)$. Since, $\Theta$ can vary between $0-180^{\circ}$ continuously, the energy deposited by the incident photon is continuous too. These energies form the Compton background in a PHS (Figure 1(c)). The maximum energy deposition through Compton scattering marks the Compton edge in the PHS. In the case of sufficiently thick (large volume) detectors, the scattered photon can undergo successive collisions eventually depositing their full energy. However, in case of thin detectors the Compton scattered photons generally leave the detector with partial energy deposition. PE events always leads to full-energy deposition and are manifested as discrete peaks in a PHS with a distribution width commensurate with the resolution of the spectrometer. Another fundamental difference between charged particle and gamma-photon interactions with matter is that charged particles of same energy have a well-defined projected range (within statistical fluctuations) for a given incident energy. The variation of the projected range with incident energy is very well defined and correlated, whereas gamma photons of the same incident energy can interact at random locations within the detector volume and lose their entire energy abruptly at the location of the interaction. The fact that the monoenergetic photons interact at different locations adds to the complexity of obtaining high resolution PHS when charge trapping (and detrapping) effects in the semiconductor come into play.

\subsection{Machine learning (ML) and convolutional neural network (CNN)}

A neural network [45] is a class of non-linear function approximators inspired by the brain. Neural networks are made up of artificial neurons, also referred to as units, and artificial synapses, also referred to as weights, that form input and output connections between neurons. Each neuron outputs a numerical value that is determined in part by the output of all of its incoming connections, the weights associated with each incoming connection, and an optional function. For example, in many applications of neural networks the output of a neuron is a sigmoidal or rectified linear function of the weighted sum of input neurons to the neuron. Neural networks are often used for function approximation in tasks involving classification and regression. The neural network is given example data as input, computes an output for this input data, and, based on the output the neural network gives and the desired output, a loss is computed. The values of the weights are adjusted to minimize this given loss function. This is typically accomplished through a process called stochastic gradient descent [46].

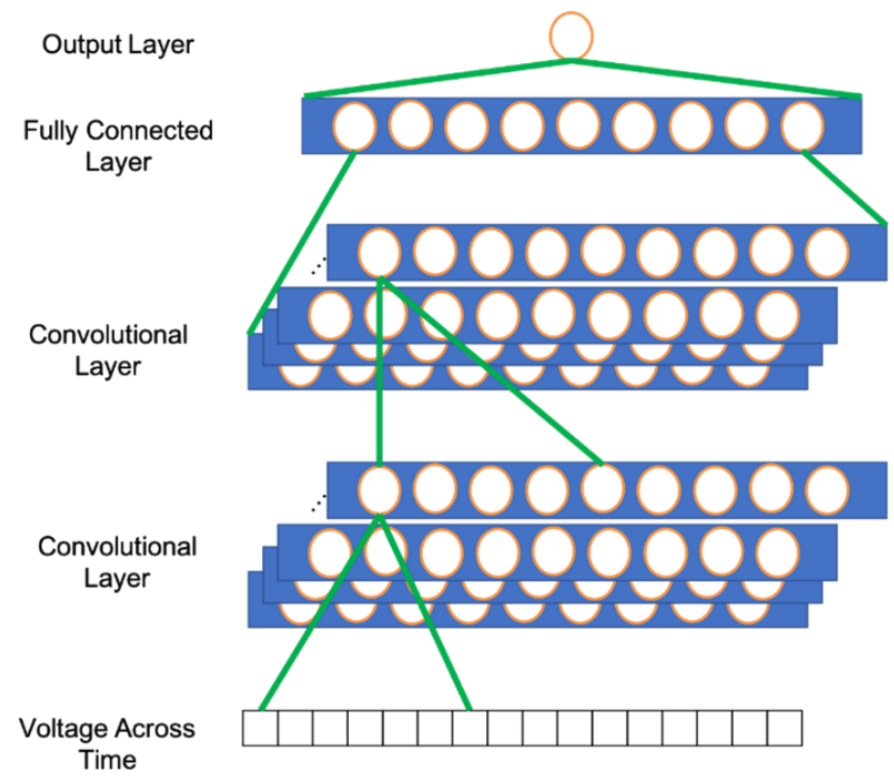

Figure 2. Schematics of the architecture of an 1D convolutional neural network.

Neural networks are often organized into groups of neurons called layers, where each layer is connected by a different set of weights. The frequently used fully connected layer is a layer where all neurons are connected to all other neurons in the 
previous layer. Structure found in data such as sound, in the one-dimensional case, and images, in the two-dimensional case, can be exploited with convolution. A convolutional neural network (CNN) employs convolution, which amounts to weight sharing, where groups of neurons in a single layer are connected by the same set of weights. Each set of weights is typically referred to as a filter and each intra-layer group of neurons is typically referred to as a channel. The stride of the convolution indicates how many neuron positions, and the weights are incremented during convolution. For example, a stride of one is a full convolution while a stride of two is a convolution that skips over one neuron each time. This will result in the subsequent layer having a dimensionality that is half that of the original layer. Figure 2 shows a visualization of a one-dimensional CNN.

\section{DATA SIMULATION AND ACQUISITION}

The common approach to training an algorithm in the field of nuclear radiation detection is to use simulated data that resemble the real-field situation. Simulated data adds to the flexibility that a trainer needs to train an algorithm. For example, noise levels affecting the signal-to-noise ratio can complicate the initial training and testing of the CNN architecture. Simulated data can be generated with the required level of noise or no noise at all. Also, there are practical impediments to obtain real data for any choice of incident radiation energy.

In the present work we used a Monte-Carlo simulation and a custom in-house code in MATLAB to generate realistic pulses that resemble the ones obtained from a real CZTS detector in a planar geometry coupled to a charge-sensitive preamplifier. As has been mentioned in Section 2.2, gamma-rays of the same energies interact at different depths within the active detector material. As a first step, the program statistically generates a set of interaction depths in the CZTS material for various energies and cross-sections obtained using NIST's XCOM web tool [47]. The program inputs the material specific parameters such as thickness of the detector, density of the detector material, and carrier mobility in the detector material. The present $\mathrm{CNN}$ has been trained separately for photoelectric (PE) and Compton scattering (CS) events. Hence, the simulation program has been designed to generate two separate files for the two types of scattering. To simulate the PE events, the program reads the above-mentioned energies and cross-sections to calculate the attenuation coefficients using the user input material density. Next, the probability of a scattering event to occur as a function of depth is determined. The second step of the program is to simulate the charge pulses that results due to the drift of the charge carriers generated by the gamma photon under the influence of the applied electric field for charge collection. The number of charge-pairs created due to an interaction is determined by the electron-hole pair creation energy (ehp), which was assumed to be same as CZT $(4.67 \mathrm{eV})$, since CZT and CZTS have similar bandgap energies $(\sim 1.6 \mathrm{eV})$. Electron and hole mobility values in CZTS were determined to be 692 and $55 \mathrm{~cm}^{2} / \mathrm{V}$-s, respectively. The mobility values have been experimentally measured using CZTS detectors fabricated in our laboratory using single crystals grown in our laboratory [25]. The mobility values have been used to calculate the drift times of the charge carriers required to drift from the point of interaction to the respective collecting electrodes. The evolution of the charge pulses is obtained by calculating the weighting potential as the charge carriers approach their respective collecting electrodes. A Gaussian noise has been embedded in the charge pulses to depict the collective noise generated from various sources such as uncertainties due to statistical nature of the nuclear events, charge creation, charge collection, and electronic noise. The SNR was varied to simulate pulses that would be obtained from spectrometers employing detectors with different energy resolutions. To generate the Compton events, additional statistical variation has been included to simulate the randomness of the relative scattering angle, which determines how much energy has been imparted to the recoiling atom (nucleus).

Figure 3 shows a few representative simulated pulse shapes obtained using the simulation code. To meet the requirement of the CNN training data size, tens of thousands of such pulses were simulated for each incident gamma energy and for each type of scattering mechanism. Each pulse has few thousand data points with a sampling interval of $10^{-8}$ sec that will result in pulse durations of tens of microseconds. Such settings resemble the experimental parameters usually set in a digital spectrometer.

To understand the effect of the variation of the signal-to noise ratio, the simulated pulses were shaped using a soft-amplifier that offers a CR-RC $\mathrm{C}^{4}$ shaping algorithm [48]. The shaped pulses were binned to generate the pulse height spectra. The width of the photopeak in the PHS were used to determine the change in the energy resolution of the spectrometer due to the change in the SNR. Figure 4 shows the PHS obtained for the case where a CZTS detector interacts with 662-keV gamma rays with different SNRs. 

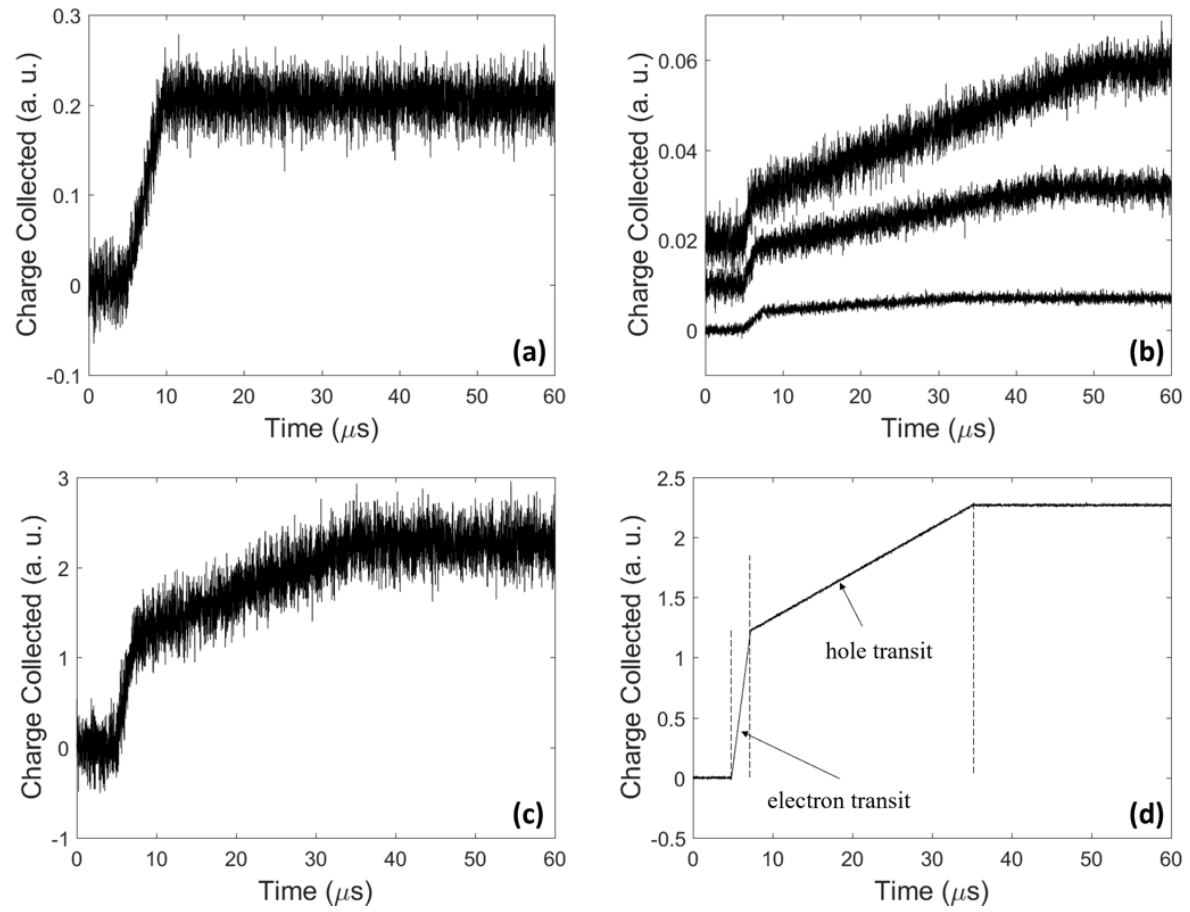

Figure 3. Simulated evolution of charge pulses for (a) Photoelectric absorption for 60-keV gamma rays; (b) Compton scattering of $60-\mathrm{keV}$ gamma interaction; (c) Photoelectric absorption of $662-\mathrm{keV}$ gamma rays with a SNR $=20$; and (d) Photoelectric absorption of $662-\mathrm{keV}$ gamma rays with a $\mathrm{SNR}=50$.
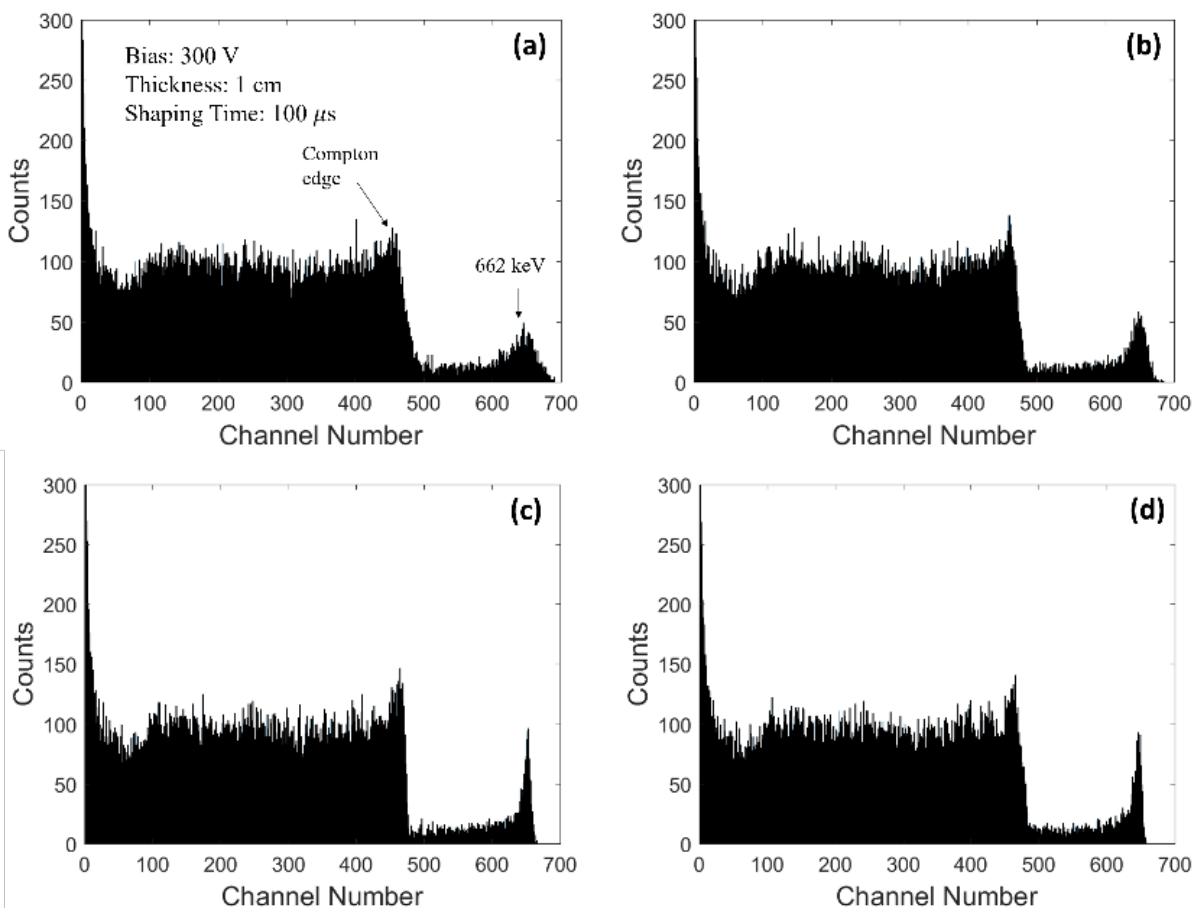

Figure 4. PHS generated using the simulated pulses with signal-to-noise ratios (a) 5, (b) 10, (c) 30, and (d) 70. 


\section{RESULTS AND DISCUSSIONS}

A simulated data file containing pulses of different energies ranging from $5 \mathrm{keV}$ to $1 \mathrm{MeV}$ was used to train the CNN algorithm [49]. The simulated data is normalized to have a mean of zero and a standard deviation of one. The input to the CNN contains 28,000 points of raw measurement. The CNN has 7 1-dimensional convolutional layers with filters of size 11. Each layer has 50 channels. The first 5 layers have a stride of 2 , and the last two have a stride of 5 . This is then followed by a fully connected layer of size 1000 and another of size 1, which represents the energy prediction. The CNN uses rectified linear activation functions [50] at all layers except the output layer, which is linear. The CNN also uses weight normalization [51] at all layers except the output layer. The CNN is trained for 1 million iterations using the ADAM optimizer with a batch size of 100 [52]. The learning rate is initialized at 0:001 and is halved if the validation accuracy does not increase within 5,000 iterations. The CNN was trained on the equivalent of $90 \%$ of the simulated data and validated on $10 \%$ of the simulated data (i.e., the energy estimation has been carried out in $0.28 \mathrm{msec}$ ). Figure 5(a) plots the predicted energy (NNet output. NNet stands for neural network) as a function of the actual energy. The average percentage error in the prediction of the gamma-ray energies have been plotted in Figure 5(b). The figure shows that, on average, the $\mathrm{CNN}$ is within $1 \%$ of the true energy for all energies and is within $0.3 \%$ of the true energy for higher energies.
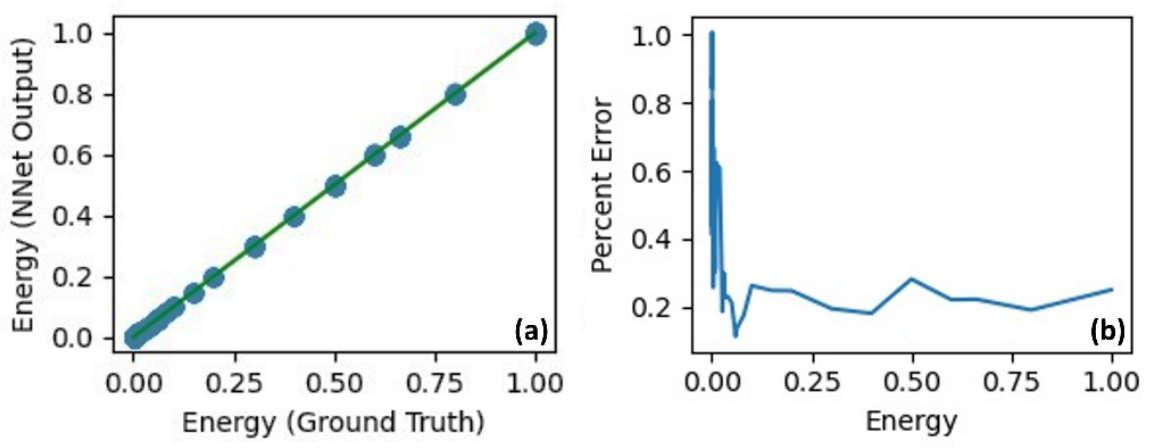

Figure 5. (a) Correlation plot of the predicted energy [NNet (Neural Network) Output] and actual energy (Ground Truth). (b) Dependence of the error of prediction on incident gamma energy.
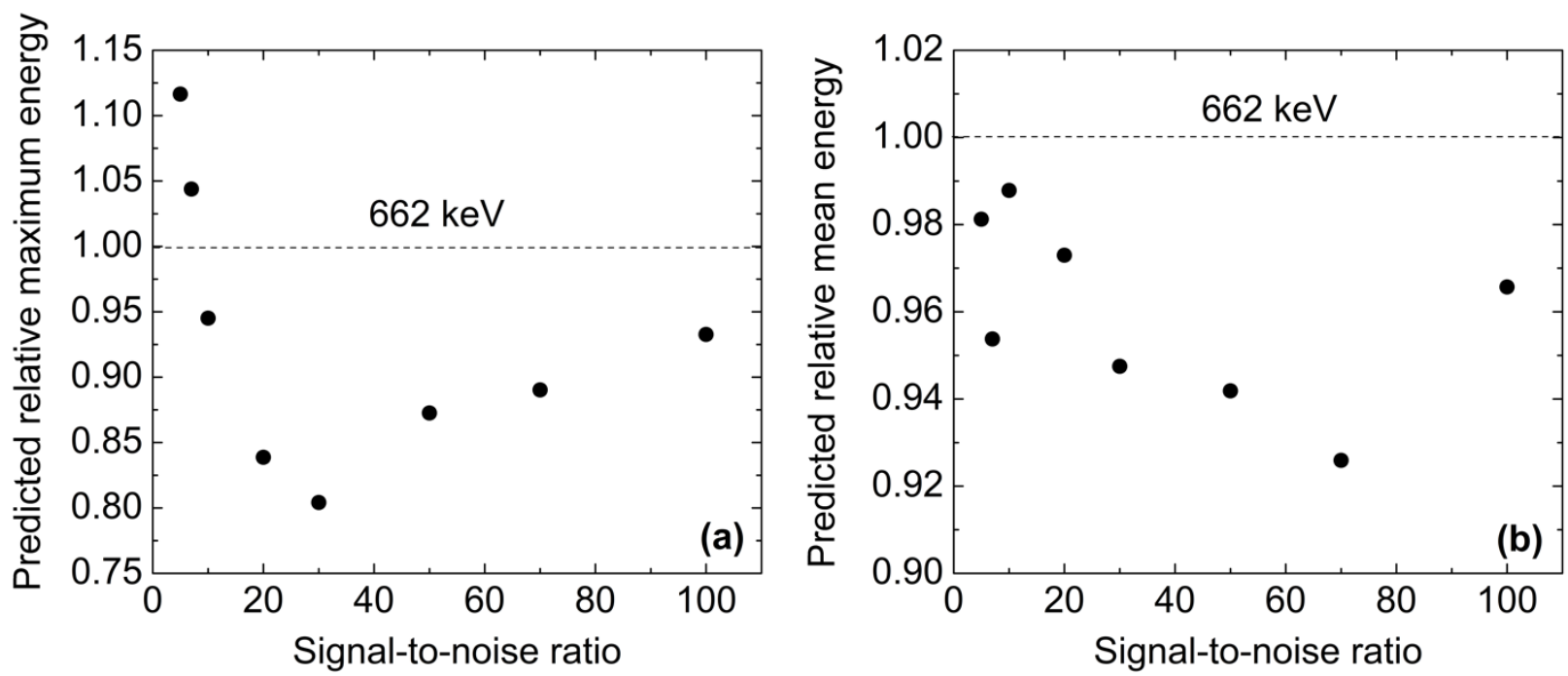

Figure 6. (a) Variation of the predicted relative maximum energy as a function of the signal-to-noise ratio obtained using a $\mathrm{PE}$ data trained CNN and (b) Variation of the predicted relative mean energy as a function of the signal-to-noise ratio obtained using a CS data trained CNN. 
We have also validated the trained $\mathrm{CNN}$ with simulated data for a single gamma-energy $(662 \mathrm{keV})$ with different noise levels. The data sets are composite, meaning they contain both the Compton and the photoelectric events randomly with a preset photopeak-to-Compton ratio. The input data were run separately through both the photoelectric and the Compton trained CNN. Figures 6 (a) \& (b) show the variation of the prediction with different signal-to-noise ratio using the photoelectric and the Compton trained $\mathrm{CNN}$, respectively. The predicted relative energy is defined as the ratio of the predicted energy to the actual energy $(662 \mathrm{keV})$. The CS trained CNN seems to perform better compared to the PE trained. The trend in the observed variation of the predicted relative energy with the signal-to-noise ratio is counterintuitive and needs further studies for appropriate explanation.

\section{SUMMARY AND CONCLUSIONS}

A convolutional neural network has been designed to identify gamma-ray energies through pattern recognition technique. The CNN has been trained using simulated data that resemble raw preamplifier pulses obtained from a CZTS detector connected to a charge sensitive preamplifier and exposed to a gamma-ray source. CZTS detector has been chosen as they are expected to be a future generation high-resolution detector for room temperature gamma-ray detection in the field of monitoring and safeguarding special nuclear materials, spent nuclear fuels, and medical imaging. The pulses have been simulated using a Monte-Carlo simulation code developed in-house. The trained CNN has been demonstrated to identify gamma energies in duration as fast as $0.28 \mathrm{msec}$. The error in the predictions of the $\mathrm{CNN}$ were found to be below $0.3 \%$ of the actual energy except at lower energies where the percentage error is below $1 \%$. The efficiency of the CNN was evaluated by varying the signal-to-noise ratio of the input signal. It was observed that the trained CNN can identify the gamma-ray energies with accuracies above $95 \%$. We plan to extend the studies by evaluating the CNN as a multi-class multi-level classification system, wherein multiple gamma energies emitted from radioactive sources comprised of multiple radioisotopes will be attempted. We also plan to develop the CNN further to identify gamma-ray energies embedded in high background radiation.

\section{ACKNOWLEDGEMENTS}

The authors acknowledge partial financial support provided by the DOE Office of Nuclear Energy's Nuclear Energy University Program (NEUP), Grant Nos. DE-AC07-051D14517 and DE-NE0008662. The work was also supported in part by the Advanced Support Program for Innovative Research Excellence-II (ASPIRE-II), \#155300-21-57381 and ASPIRE-IIB, \#155300-21-56895 of the University of South Carolina (UofSC), Columbia, USA.

\section{REFERENCES}

[1] Mariscotti, M. A., "A method for automatic identification of peaks in the presence of background and its application to spectrum analysis," Nucl. Instrum. Meth. 50(2), 309-320 (1967).

[2] Phillips, G. W. and Marlow, K. W., "Automatic analysis of gamma-ray spectra from germanium detectors," Nucl. Instrum. Meth. 137(3), 525-536 (1976).

[3] Cherepy, N. J., Payne, S. A., Sturm B. W., Drury, O. B., O'Neal, S. P., Thelin, P. A., Shah, S. K., Hawrami, R., Momayezi, M., Hurst, B., Burger, A., Wiggins, B., Bhattacharya, P., Boatner L. A. and Ramey, J. O., "Instrument development and gamma spectroscopy with strontium iodide," IEEE Trans. Nucl. Sci. 60(2), 955 - 958 (2013).

[4] Abbene, L., Principato, F., Gerardi, G., Buttacavoli, A., Cascio, D., Battelli, M., Amadè, N. S., Seller, P., Veale, M. C., Fox, O., Sawhney, K., Zanettini, S., Tomarchio E. and Zappettini, A., "Room-temperature X-ray response of cadmium-zinc-telluride pixel detectors grown by the vertical Bridgman technique," J. Synchrotron Radiat. 27(2), 319-328 (2020).

[5] Dewji, S. A., Croft, S. and Hertel, N. E., "Sensitivity analysis of high-resolution gamma-ray detection for safeguards monitoring at natural uranium conversion facilities," Nucl. Instrum. Meth. Phys. Res. A 848, 153-161 (2017).

[6] Durham, J. M., Poulson, D., Bacon, J., Chichester, D. L., Guardincerri, E., Morris, C. L., Plaud-Ramos, K., Schwendiman, W., Tolman, J. D. and Winston, P., "Verification of spent nuclear fuel in sealed dry storage casks via measurements of cosmic-ray muon scattering," Phys. Rev. Appl. 9, 044013-1-8 (2018). 
[7] Çınar, H., Altundaş, S., Ersoy, E., Bak, K. and Bayrak, N., "Application of two geophysical methods to characterize a former waste disposal site of the Trabzon-Moloz district in Turkey," Environ. Earth Sci. 75, 52-1-16 (2015).

[8] Briganti, A., Tuccimei, P., Voltaggio, M., Carusi, C., Galli, G. and Lucchetti, C., "Assessing methyl tertiary butyl ether residual contamination in groundwater using radon," Appl. Geochemistry 116, 104583-1-9 (2020).

[9] Shizuma, K., Fujikawa, Y., Kurihara, M. and Sakurai, Y., "Identification and temporal decrease of ${ }^{137} \mathrm{Cs}$ and ${ }^{134} \mathrm{Cs}$ in groundwater in Minami-Soma City following the accident at the Fukushima Dai-ichi nuclear power plant," Environ. Pollut. 234, 1-8 (2018).

[10] Ambrosino, F., De Cesare, W., Roca, V. and Sabbarese, C., "Mathematical and geophysical methods for searching anomalies of the Radon signal related to earthquakes," J. Phys. Conf. Ser. 1226, 012025-1-5 (2018).

[11] Morales-Simfors, N., Wyss, R. A. and Bundschuh, J., "Recent progress in radon-based monitoring as seismic and volcanic precursor: A critical review," Crit. Rev. Env. Sci. Tec. 50(10), 979-1012 (2019).

[12] Boller, P., Zylstra, A., Neumayer, P., Bernstein, L., Brabetz, C., Despotopulos, J., Glorius, J., Hellmund, J., Henry, E. A., Hornung, J., Jeet, J., Khuyagbaatar, J., Lens, L., Roeder, S., Stoehlker, T., Yakushev, A., Litvinov, Y. A., Shaughnessy, D., Bagnoud, V., Kuehl, T. and Schneider, D. H. G., "First on-line detection of radioactive fission isotopes produced by laser-accelerated protons," Sci. Rep. 10, 17183-1-9 (2020).

[13] Johns, P. M. and Nino, J. C., "Room temperature semiconductor detectors for nuclear security," J. Appl. Phys. 126, 040902-1-20 (2019).

[14] Schlesinger, T. E., Toney, J. E., Yoon, H., Lee, E. Y., Brunett, B. A., Franks, L. and James, R. B., "Cadmium zinc telluride and its use as a nuclear radiation detector material," Mater. Sci. Eng. R Rep. 32, 103-189 (2001).

[15] Bell, S. J., Baker, M. A., Duarte, D. D., Schneider, A., Seller, P., Sellin, P. J., Veale, M. C. and Wilson, M. D., "Performance comparison of small-pixel CdZnTe radiation detectors with gold contacts formed by sputter and electroless deposition," J. Instrum. 12, 6015-1-10 (2017).

[16] Sajjad, M., Chaudhuri, S. K., Kleppinger, J. W. and Mandal, K. C., "Growth of large-area $\mathrm{Cd}_{0.9} \mathrm{Zn}_{0.1} \mathrm{Te}$ single crystals and fabrication of pixelated guard-ring detector for room-temperature $\gamma$-ray detection," IEEE Trans. Nucl. Sci. 67(8), 1946-1951 (2020).

[17] Chaudhuri, S. K., Kleppinger, J. W., Karadavut, O. F., Nag, R. and Mandal, K. C., "Quaternary semiconductor Cd ${ }_{1-}$ ${ }_{x} \mathrm{Zn}_{\mathrm{x}} \mathrm{Te}_{1-\mathrm{y}} \mathrm{Se}_{\mathrm{y}}$ for high-resolution, room-temperature gamma-ray detection," Crystals 11, 827-1-22 (2021).

[18] Roy, U. N., Camarda, G. S., Cui, Y. and James, R. B., "Advances in CdZnTeSe for radiation detector applications," Radiation 1, 123-130 (2021).

[19] Franc, J., Höschl, P., Belas, E., Grill, R., Hlídek, P., Moravec, P. and Bok, J., "CdTe and CdZnTe crystals for room temperature gamma-ray detectors," Nucl. Instrum. Meth. Phys. Res. A 434(1), 146-151 (1999).

[20] Chaudhuri, S. K., Lohstroh, A., Nakhostin, M. and Sellin, P. J., "Digital pulse height correction in $\mathrm{HgI}_{2} \gamma$-ray detectors," J. Instrum. 7, T04002-1-13 (2012).

[21] He, Y., Matei, L., Jung, H. J., McCall, K. M., Chen, M., Stoumpos, C. C., Liu, Z., Peters, J. A., Chung, D. Y., Wessels, B. W., Wasielewski, M. R., Dravid, V. P., Burger, A. and Kanatzidis, M. G., "High spectral resolution of gamma-rays at room temperature by perovskite $\mathrm{CsPbBr}_{3}$ single crystals," Nat. Commun. 9, 1609-1-8 (2018).

[22] Roy, U. N., Camarda, G. S., Cui, Y., Gul, R., Yang, G., Zazvorka, J., Dedic, V., Franc, J. and James, R. B., "Evaluation of CdZnTeSe as a high-quality gamma-ray spectroscopic material with better compositional homogeneity and reduced defects," Sci. Rep. 9(1), 7303-1-7 (2019).

[23] Roy, U. N., Camarda, G., Cui, Y., Yang, G. and James, R. B., "Impact of selenium addition to the cadmium-zinc-telluride matrix for producing high energy resolution X-and gamma-ray detectors," Sci. Rep. 11, 10338-1-10 (2021).

[24] Chaudhuri, S. K., Sajjad, M. and Mandal, K. C., "Pulse-shape analysis in $\mathrm{Cd}_{0.9} \mathrm{Zn}_{0.1} \mathrm{Te}_{0.98} \mathrm{Se}_{0.02}$ room-temperature radiation detectors," Appl. Phys. Lett. 116, 162107-1-5 (2020).

[25] Chaudhuri, S. K., Sajjad, M., Kleppinger, J. W. and Mandal, K. C. "Charge transport properties in CdZnTeSe semiconductor room-temperature $\gamma$-ray detectors," J. Appl. Phys. 127, 245706-1-8 (2020).

[26] Chaudhuri, S. K., Sajjad, M., Kleppinger, J. W. and Mandal, K. C., "Correlation of space charge limited current and $\gamma$-ray response of $\mathrm{Cd}_{\mathrm{x}} \mathrm{Zn}_{1-\mathrm{x}} \mathrm{Te}_{1-\mathrm{y}} \mathrm{Se}_{\mathrm{y}}$ room-temperature radiation detectors," IEEE Electron Dev. Lett. 41(9), 1336$1339,(2020)$.

[27] Hwang, S., Yu, H., Bolotnikov, A. E., James, R. B. and Kim, K., "Anomalous Te inclusion size and distribution in CdZnTeSe," IEEE Trans. Nucl. Sci. 66(11), 2329 - 2332 (2019).

[28] Pipek, J., Betušiak, M., Belas, E., Grill, R., Praus, P., Musiienko, A., Pekarek, J., Roy, U. N. and James, R. B., "Charge transport and space-charge formation in $\mathrm{Cd}_{1-\mathrm{x}} \mathrm{Zn}_{\mathrm{x}} \mathrm{Te}_{1-\mathrm{y}} \mathrm{Se}_{\mathrm{y}}$ radiation detectors," Phys. Rev. Appl. 15, 054058-1-8 (2021). 
[29] Yakimov, A., Smith, D., Choi, J. and Araujo, S., "Growth and characterization of detector-grade CdZnTeSe by horizontal Bridgman technique," Proc. SPIE 1114, 11141N-1-7 (2019).

[30] Silagadze, Z. K., "A new algorithm for automatic photopeak searches," Nucl. Instrum. Meth. Phys. Res. A 376(3), 451-454 (1996).

[31] Cheknov, S. V., Childers, J. T., Proudfoot, J., Wang, R. and Frizzell, D., "Precision searches in dijets at the HL-LHC and HE-LHC," J. Instrum. 13, P05022-1-22 (2018).

[32] Schug, D., Wehner, J., Goldschimdt, B., Lerche, C., Dueppenbecker, P. M., Hallen, P., Weissler, B., Gebhardt, P., Kiessling, F. and Schulz, V., "Data processing for a high-resolution preclinical PET detector based on Philips DPC digital SiPMs," IEEE Trans. Nucl. Sci. 62(3), 669-678 (2015).

[33] Abdel-Aal, R. E. and Al-Haddad, M. N. "Determination of radioisotopes in gamma-ray spectroscopy using abductive machine learning," Nucl. Instrum. Meth. Phys. Res. A 391(2), 275-288 (1997).

[34] Vigneron, V., Morel, J., Lepy, M. C. and Martinez, J. M., "Statistical modelling of neural networks in gspectrometry," Nucl. Instrum. Meth. Phys. Res. A 369(2-3), 642-647 (1996).

[35] Ford, W. P., Hague, E., McCullough, T., Moore, E. and Turk, J., "Threat determination for radiation detection from the Remote Sensing Laboratory," Proc. SPIE 10644, 106440G (2018).

[36] Bukartas, A., Finck, R., Wallin, J. and Rääf, C. L., "A Bayesian method to localize lost gamma sources," Appl. Rad. Isot. 145, 142-147 (2019).

[37] Kamuda, M., Zhao, J. and Huff, K., "A comparison of machine learning methods for automated gamma-ray spectroscopy," Nucl. Instrum. Meth. Phys. Res. A, 954, 161385-1-6 (2020).

[38] Almaniotis, M., Mattingly, J. and Tsoukalas, L. H., "Kernel-based machine learning for background estimation of NaI low-count gamma-ray spectra," IEEE Trans. Nucl. Sci. 60(3), 2209-2221 (2013).

[39] Duda R. and Hart, P. [Pattern Recognition and Scene Analysis], Wiley, New York, (1973).

[40] Wasserman, P. D., [Neural Computing: Theory and Practice], Van Nostrand Reinhold, New York (1989).

[41] Breiman, L., Friedman, J., Olshen, R. and Stone, C., [Classification and Regression Trees], Wadsworth, Belmont, CA (1984).

[42] Montgomery, G. J. and Drake, K. C., "Abductive networks," Proc. SPIE 1294 56-64 (1990).

[43] Knoll, G. F., [Radiation Detection and Measurements], John Wiley, Hoboken, NJ (2010).

[44] Nakhostin, M., Signal Processing for Radiation Detectors, Hoboken, NJ, Wiley (2018).

[45] Schmidhuber, J., "Deep learning in neural networks: An overview," Neural Netw. 61, 85-117 (2015).

[46] Rumelhart, D. E. and McClelland, J. L., "Learning internal representations by error propagation," in [Parallel Distributed Processing: Explorations in the Microstructure of Cognition: Foundations], MIT Press, Cambridge, MA, 318-362 (1987).

[47] NIST, XCOM, Webtool, https://physics.nist.gov/PhysRefData/Xcom/html/xcom1.html.

[48] Nakhostin, M., "Recursive algorithms for real-time CR-(RC) digital pulse shaping," IEEE Trans. Nucl. Sci. 58(5), 2378-2381 (2011).

[49] LeCun, Y., Boser, B., Denker, J. S., Henderson, D., Howard, R. E., Hubbard, W. and Jackel, L. D., "Backpropagation applied to handwritten zip code recognition," Neural Comput. 1(4), 541-551 (1989).

[50] Jarrett, K., Kavukcuogulo, K., Ranzato, M. and LeCun, Y., "What is the best multi-stage architecture for object recognition?" in 2009 IEEE 12th International Conference on Computer Vision, Kyoto, Japan (2009).

[51] Salimans, T. and Kingma, D. P. "Weight normalization: A simple reparameterization to accelerate training of deep neural networks," Advances in Neural Information Processing Systems 29, 901-909 (2016).

[52] Kingma, D. P. and Ba, J., "Adam: A method for stochastic optimization," arXiv:1412.6980v9 (2017). 